\title{
Emergence of a confined state in a weakly bent wire
}

\author{
Er'el Granot* \\ School of Physics and Astronomy, Raymond and Beverly Sackler Faculty of Exact Sciences, Tel-Aviv University, 69978 Tel \\ Aviv, Israel
}

(October 30, 2018)

\begin{abstract}
In this paper we use a simple straightforward technique to investigate the emergence of a bound state in a weakly bent wire. We show that the bend behaves like an infinitely shallow potential well, and in the limit of small bending angle $(\varphi \ll 1)$ and low energy the bend can be presented by a simple $1 \mathrm{D}$ delta function potential: $V(x)=-\left(2 \sqrt{c_{b}} \varphi^{2}\right) \delta(x)$ where $c_{b} \cong 2.1$
\end{abstract}

PACS: 72.10.Fk, 73.20.Dx, and 03.65.Ge

It was well known for decades that the electric transmission of a quantum wire (and, in general, any waveguide) is strongly affected by the wire's boundaries' topology. Nevertheless, since 1989 many researchers have validated a surprising finding. Exner and Seba [1] were the first to show that a smoothly curved waveguide holds a confined eigenstate, whose energy is lower than the waveguide's cut-off energy. This bound state exists even when there is no change in the waveguide's width. Avishai and coworkers [2] have used an elegant variational proof not only to show that a bound state exists in a broken wire, but also to evaluated its eigenvalue in the limit of small bending angle. Later on Goldstone and Jaffe [3] generalized these findings and proved that any wire of constant width with any bend will support at least one bound state (provided the wire eventually straightens)

While the presence of such bound states was well proven by many authors [4]- [8] their existence is still a puzzling problem.

Carini et al. [7] suggested a qualitative explanation as to why bends (and of course bulges) produce an effective attraction and therefore a bound state. By substituting in the Schrdinger equation a trial wave function for the lowest bound state the problem is reduced to a $1 \mathrm{D}$ one. They showed that in this case the bend can be regarded as an attractive (it is always negative) 1D potential. In 1D, such a potential always has a bound state.

Their qualitative description holds only in the adiabatic approximation, i.e., when the wire's curvature is always small and slowly changing. In the extreme case, where the bend occurs at a single point (like the one discussed by Avishai et al. [2], see Fig.1), such reasoning (which cannot be applied) would yield an effective potential well, whose length is proportional to the bending angle, $\varphi$ (note, that the potential depth is almost independent of $\varphi$ ). For such a potential well the lowest bound state eigen energy goes like $-\varphi^{2}$.

Similarly, according to Sols and Macucci [4], the bend

erel.g@chilight.com can be regarded as a small resonator whose effective width is slightly larger than that of the waveguide in which it is introduced. This larger effective width accounts for the lower minimum energy for propagation, while the effective length of the resonator is proportional to the bend's angle. Such a simplified qualitative description again predicts bound eigen energy, which is proportional to $-\varphi^{2}$. These evaluations, however contradicts the result of ref. [2], in which it was proven that the eigen energy is quartic with respect to $\varphi$ (for small bending angles). The quartic dependence also appears in mildly curved wire (see ref. [9] and references therein).

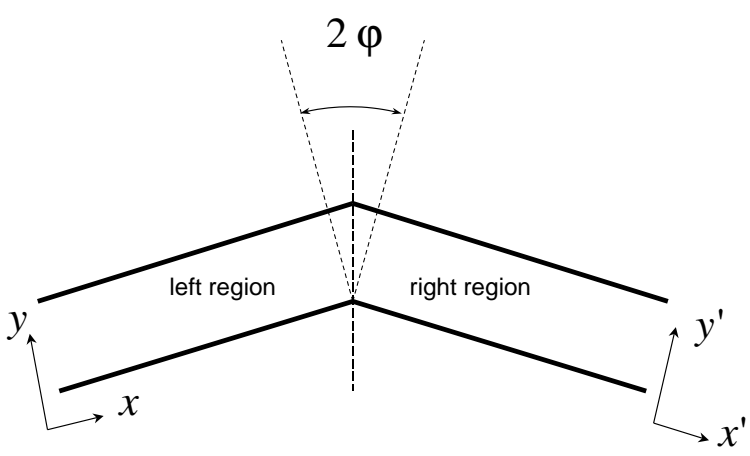

FIG. 1. An illustration of the bent wire.

The discrepancy appears since in the regime of weakly bending wire (i.e., small bending angle) these simplified pictures (the cavity picture, for example) cannot be applied. For example, in the cavity picture most of the wavefunction will be distributed outside the cavity.

In this short paper we show that in this case the bend behaves like an infinitely shallow well(ISW), and that in the low energy regime it can be replaced by a delta function potential in a $1 \mathrm{D}$ wire 10 , and therefore cannot be presented by a simple $2 \mathrm{D}$ cavity. 
There are several methods to calculate the boundstates eigen energies of a bent wire (or waveguide). The most direct (and probably the most common) method is to divide the wire into three parts: before the bend (a perfect lead), the bent region, and after the bend (a perfect lead). This method is usually used for a circular bend (or for one at right angles), and thus, the complete set of wave functions is well known both in the bending region and in the perfect leads. Thus, the solution is straightforward after matching these solutions at the different regions' boundaries.

This method was carried out by Schult et al [5] to calculate the eigen energies of an electron caught at the intersection of two narrow (but totally diagonal) channels; Sols and Macucci [4] and Lent [6] used this method to calculate the transmission through a circular bend. It has also been used to calculate multiple bound states in a sharply bent waveguide by Carini et al [7], and in (long) circular bends by Lin and Jaffe [8].

A similar method is to use the Green function formalism: this approach was taken by Goldstone and Jaffe [3], again for a right angle (but rectangular) bend.

These two methods work very well for relatively large bending angles. However, for low bending angles, they are not very efficient, since they require extremely high accuracy of the solutions of the Bessel functions. Since the difference between the bound-state eigen energy and the cut-off (threshold) energy is proportional to $\varphi^{4}$ ( [2], [9]), then even for $\varphi=10^{-2}$ (not to mention smaller angles), the calculations require an accuracy (for the Bessel function zeros) which is higher than $10^{-9}$.

A third popular method of calculating the bound states eigen energies is to discretize the wave function over the entire 2D volume, and either by iteration [5] or by a similar relaxation method [7], to calculate the low-lying eigenvalues and eigenfunctions of the problem.

Again, this method cannot be used in the small bending angle regime since this would require extremely large matrices. For example, in the case $\varphi=10^{-2}$, the wave function will decay within a distance $10^{4}$ times larger than the wire's width. Therefore, in order to obtain the required accuracy in the transversal direction (i.e., the wire's width), the matrix will be too large to handle.

The best (and most elegant) method to calculate the eigen bound state energy is the variational one (which was used in ref. [2]). However, this method cannot be applied to the scattering case.

Hence, we use a slightly different and simpler approach. We do not divide the wire into three regions but rather into only two, and the only matching of the wave function is carried out at the bending axis. Therefore, no Bessel functions are required, and much higher accuracy can be achieved. Moreover, we match directly the wave function, and therefore no overlapping integrals are necessary, which makes it a very simple technique.
The geometry of the problem is illustrated in Fig.1. The stationary-state Schrödinger equation reads

$$
\nabla^{2} \psi(\mathbf{r})+[\omega-V(\mathbf{r})] \psi(\mathbf{r})=0
$$

(again we use the units $\hbar=2 m=1$ ). $V$ is the potential of the wire's walls (i.e., $V=0$ inside the wire and $V=\infty$ on the outside) and $\omega$ is the particle's energy.

Except for the bend, the system geometry is very simple; therefore, the space can be divided into two regions: before the bend (say, left region) and beyond it (say, right region). To simplify the notations, we use different axes in each region: $(\mathrm{x}, \mathrm{y})$ and $\left(\mathrm{x}^{\prime}, \mathrm{y}^{\prime}\right)$, respectively (see Fig.1).

Should a bound state exists, it can be presented in the following way [1]:

$$
\begin{array}{ll}
\psi_{B}^{L}(x, y)=\sum_{n=1}^{\infty} d_{n} \sin \left(k_{n} y\right) e^{\alpha_{n} x} & \text { for the left region } \\
\psi_{B}^{R}\left(x^{\prime}, y^{\prime}\right)=\sum_{n=1}^{\infty} d_{n} \sin \left(k_{n} y^{\prime}\right) e^{-\alpha_{n} x^{\prime}} & \text { for the right region }
\end{array}
$$

the subscript "B" represents bound state, and the superscripts "L" and "R" designate the left and right regions, respectively, and

$$
k_{n} \equiv n \pi
$$

and

$$
\alpha_{n} \equiv \sqrt{(n \pi)^{2}-\omega}
$$

The strategy is the following [1]: $\psi_{B}^{L}$ is a solution in the entire left region. We don't say yet that this is the right one, but this is definitely a solution, because it solves the Schrödinger equation in the entire left region, and it agrees with the boundary conditions of this region (except, for the moment, the one at $x \geq 0$ ). The same argument applies for $\psi_{B}^{R}$ : it solves the Schrödinger equation and maintains the boundary conditions in the entire right region. Therefore it is a solution in that entire region.

Now, we need to find the right coefficients $\left(d_{n}\right)$, which will take care of the boundary condition at the break, i.e., the continuity of the wave function and its derivative at the break boundary. In order to do so, we match the wave function and its derivative at $N$ different points on this line, then we take the limit $N \rightarrow \infty$ and show that the solution (and the coefficients) converge to a specific function.

Let us define a new set of coordinates:

$$
\left(\begin{array}{l}
\xi \\
\eta
\end{array}\right) \equiv\left(\begin{array}{cc}
\cos \varphi & \sin \varphi \\
-\sin \varphi & \cos \varphi
\end{array}\right)\left(\begin{array}{l}
x \\
y
\end{array}\right)=\left(\begin{array}{cc}
\cos \varphi & -\sin \varphi \\
\sin \varphi & \cos \varphi
\end{array}\right)\left(\begin{array}{l}
x^{\prime} \\
y^{\prime}
\end{array}\right)
$$

Then the wave function on the left side of the bend is: 


$$
\sum_{n=1}^{\infty} d_{n} \sin \left[k_{n}(\xi \sin \varphi+\eta \cos \varphi)\right] \exp \left[\alpha_{n}(\xi \cos \varphi-\eta \sin \varphi)\right]
$$

and on the right side:

$$
\sum_{n=1}^{\infty} d_{n} \sin \left[k_{n}(-\xi \sin \varphi+\eta \cos \varphi)\right] \exp \left[-\alpha_{n}(\xi \cos \varphi+\eta \sin \varphi)\right.
$$

With these notations in mind, the matching of the wave function should take place at $\xi=0$.

Limiting the calculations to $N$ modes, gluing of the wave function derivative at $\xi=0$, i.e. requiring that $\partial \psi_{B} /\left.\partial \xi\right|_{\xi=0}=0$, leads to a single equation with $N$ variables. To solve them, we quantize $\eta[11$ :

$$
\eta_{m} \equiv \frac{m-1}{(N-1) \cos \varphi}
$$

for $1 \leq m \leq N$.

(these are the $N$ points were the matching takes place).

The prescribed substitution solves this problem: $N$ variables and $N$ equations, which can be written

$$
\sum_{n=1}^{N} M_{n m} t_{n}=0
$$

where

$$
\begin{gathered}
M_{n m} \equiv-\left[M_{n m}^{1}+M_{n m}^{2}\right] \exp \left(-\alpha_{n} \eta_{m} \sin \varphi\right) . \\
M_{n m}^{1} \equiv k_{n} \sin \varphi \cos \left(k_{n} \eta_{m} \cos \varphi\right), \text { and } M_{n m}^{2} \equiv \\
\alpha_{n} \cos \varphi \sin \left(k_{n} \eta_{m} \cos \varphi\right) .
\end{gathered}
$$

Clearly, a solution (a bound state) exists only when the matrix determinant vanishes:

$$
\left|M_{n m}\right|=0 \text {. }
$$

Solving eq.11 numerically for $N \rightarrow \infty$ one finds that a confined solution exists and converges to

$$
\omega_{b} \rightarrow \omega_{0} \equiv \pi^{2}-c_{b} \varphi^{4}
$$

where the proportionality constant converges to the theoretical value [2]

$$
c_{b} \rightarrow 2.10 \ldots
$$

Now, if our assumption is correct, and the bend can be presented as an ISW in a 1D system in the limits $\varphi \rightarrow 0$ and $\omega-\pi^{2} \rightarrow 0$ then, it can be replaced by the following $1 \mathrm{D}$ point potential [10] (in a $1 \mathrm{D}$ wire)

$$
V(x)=-\left(2 \sqrt{c_{b}} \varphi^{2}\right) \delta(x)
$$

and the 1D transmission is obtained in straightforward fashion:

$$
t=\frac{1}{1-i \sqrt{c_{b}} \varphi^{2} / \Delta}
$$

where

$$
\Delta \equiv \sqrt{\omega-\pi^{2}}
$$

In order to show that this 1D approximation is accu) rate for the limits $\varphi, \Delta \rightarrow 0$, we will evaluate the transmission in the direct approach.

Assume that the incident wave from $x=-\infty$ is

$$
\psi_{i n c}(x, y)=\sum_{n=1}^{\infty} \sin \left(k_{n} y\right)\left[a_{n} \exp \left(i \widetilde{k}_{n} x\right)+r_{n} \exp \left(-i \widetilde{k}_{n} x\right)\right]
$$

while the transmitted one $\left(x^{\prime} \rightarrow \infty\right)$ is

$$
\psi_{\text {tran }}\left(x^{\prime}, y^{\prime}\right)=\sum_{n=1}^{\infty} t_{n} \sin \left(k_{n} y^{\prime}\right) \exp \left(i \widetilde{k}_{n} x^{\prime}\right)
$$

where $a_{n}, r_{n}$ and $t_{n}$ are the incident, reflected and transmitted coefficients respectively [note that if $a_{n}=$ $\delta_{n 1}$ then $t($ eq. 15$) \simeq t_{1}($ eq. 17$\left.)\right] ; \quad k_{n} \equiv n \pi$, and $\widetilde{k}_{n} \equiv$ $\sqrt{\omega-(n \pi)^{2}}$ (i.e., $\left.i \widetilde{k}_{n}=i \alpha_{n}\right)$.

After transforming to the new coordinates (5) and solving by following ref. [11] we obtain the transmission coefficient as a function of the bending angle for a given set of coefficients $a_{n}$.

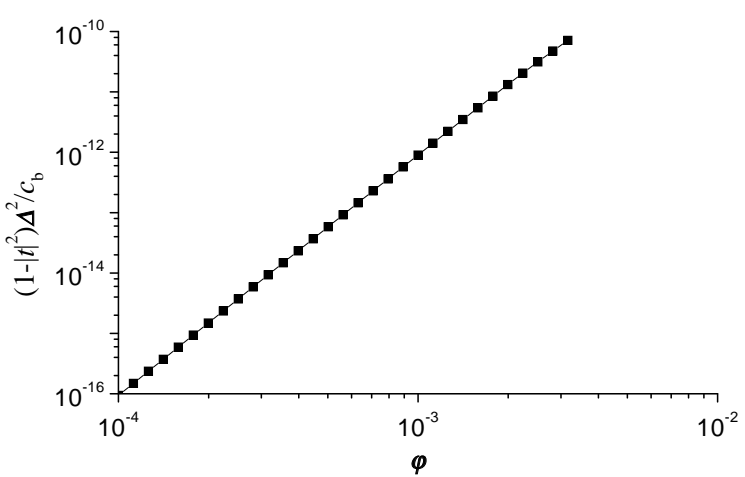

FIG. 2. Plot of the normalized reflection $\left(1-\left|t_{1}\right|^{2}\right) \Delta^{2} / c_{b}$ as a function of the bend angle $\varphi$. This plot validates the approximation of eq 18 .

The plot of $1-\left|t_{1}\right|^{2}$ as a function of the bend angle is shown in Fig.3. As can be seen from this figure, eq.15, which predicts

$$
1-\left|t_{1}\right|^{2} \sim c_{b} \varphi^{4} / \Delta^{2}
$$

(for small angles), is a very good approximation.

Hence, in the energy regime $c_{b} \varphi^{4} / \Delta^{2} \ll 1$, the scattering wave function can be reduced to a $1 \mathrm{D}$ scattering problem via the following separation of coordinates 


$$
\psi(\widetilde{x}, \widetilde{y}) \simeq \sin (\pi \widetilde{y}) \psi^{1}(\widetilde{x})
$$

where

$$
\widetilde{x}, \widetilde{y}=\left\{\begin{array}{c}
x, y, \text { for } x<0 \\
x^{\prime}, y^{\prime} \text { for } x^{\prime}>0
\end{array}\right\}
$$

and $\psi^{1}$ obeys the $1 \mathrm{D}$ Schrödinger equation

$$
-\frac{\partial \psi^{1}}{\partial \widetilde{x}^{2}}-\left(2 \sqrt{c_{b}} \varphi^{2}\right) \delta(\widetilde{x}) \psi^{1}=\Delta^{2} \psi^{1} .
$$

Eq.19 also predicts the bound eigenstate with high accuracy:

$$
\psi_{B} \simeq \sin (\pi \widetilde{y}) \exp \left(-\sqrt{c_{b}} \varphi^{2}|\widetilde{x}|\right)
$$

Clearly, this approximation is accurate for $|\widetilde{x}| \rightarrow \infty$.

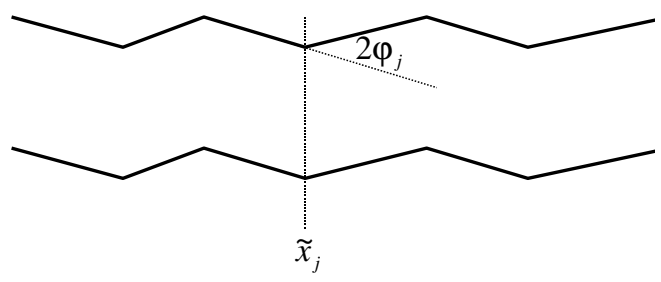

FIG. 3. Wire with rough boundaries can be presented as a wire with multiple bends

Equation 21 can easily be generalized to a wire with an arbitrary number of bends (i.e., a rough boundaries wire, see fig.3). Such a wire with rough boundaries can be presented by

$$
-\frac{\partial \psi^{1}}{\partial \widetilde{x}^{2}}-2 \sqrt{c_{b}} \sum_{j=1}^{N} \varphi_{j}^{2} \delta\left(\widetilde{x}-\widetilde{x}_{j}\right) \psi^{1}=\Delta^{2} \psi^{1} .
$$

Before summarizing, it may be of interest to compare eq.18, i.e., the low energy scattering over the bend, to scattering over a point impurity [12]: when the impurity is located a distance $\varepsilon$ from the wire's boundary the wire's transmission should hold the relation

$$
1-\left|t_{1}\right|^{2} \sim c_{i} \varepsilon^{4} / \Delta^{2}
$$

where the impurity's parameters are manifested in the coefficient $c_{i}$.

To summarise, in this paper we investigated the emergence of a bound state in a bent wire. It was shown that in the limit of small bending angle and low energy the system can be reduced to a 1D scattering problem, where the bend acts as a delta function potential, i.e., $V(x)=-\left(2 \sqrt{c_{b}} \varphi^{2}\right) \delta(x)$.

I would like to thank prof. Mark Azbel and prof. Yshai Avishai for enlightening discussions.

[1] P. Exner, and P. Seba, J. Math. Phys. 30, 2574 (1989)

[2] Y. Avishai, D.Bessis, B.G. Giraud, and G. Mantica, Phys. Rev. B 44, 8028 (1991)

[3] J. Goldstone and R.L. Jaffe, Phys. Rev. B 45, 14100 (1992)

[4] F. Sols and M. Macucci, Phys. Rev. B 41, 11887 (1990)

[5] R.L. Schult, D.G. Ravenhall and H.W. Wyld, Phys. Rev. B 39, 5476 (1989)

[6] C.S. Lent, Appl. Phys. Lett. 56, 2554 (1990)

[7] J.P. Carini, J.T. Londergan, K. Mullen and D.P. Murdock, Phys. Rev. B 48, 4503 (1993)

[8] K. Lin and R.L. Jaffe, Phys. Rev. B 54, 5750 (1996)

[9] P. Duclos and P. Exner, Rev. Math. Phys. 7, 73 (1995)

[10] If the width $W$ and depth $v$ of an arbitrary well satisfy $|v| \ll W^{-2}$ the well is regarded as a shallow one. Therefore, a delta function is an ideal ISW in 1D.

[11] E. Granot, Phys. Rev. B 60, 14172 (1999)

[12] E. Granot, Phys. Rev. B 60, 10664 (1999);B 61, 11078 (2000). 